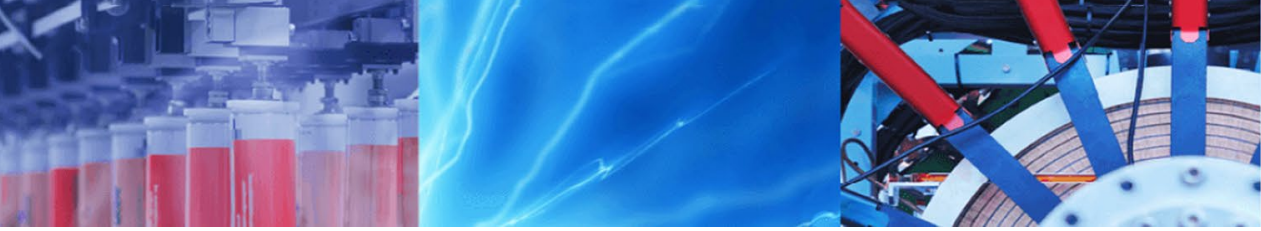

Research Article

\title{
Effect of extrusion on viscoelastic slurry 3D print quality: numerical analysis and experiment validation
}

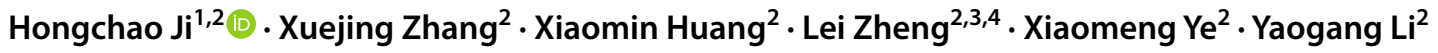

(c) Springer Nature Switzerland AG 2019

\begin{abstract}
As a new manufacturing technology, three-dimensional (3D) printing is being applied gradually to all walks of life. 3D printing ceramic materials can solve the problem of hard and brittle materials in shape processing. In this study, precursor ceramic parts were prepared based on the technology of fusion deposition molding. To attain high surface accuracy of ceramic green samples, 3D printer plunger and screw extruders were applied for printing and for comparing the printing results. Two types of slurry extruder were simulated and analyzed. The determined slurry extrusion device was optimized. Results show that the quality of the green sample obtained by the screw extruder is significantly better than that obtained by the plunger extruder. The optimized 3D printer can finish printing the ceramic sample efficiently with the required printing precision.
\end{abstract}

Keywords Precursor ceramic materials - 3D printer - Extrusion process of additive manufacturing technology - Screw extruder $\cdot$ Plunger extruder $\cdot$ Print quality comparison

\section{Introduction}

3D printing is known as an additive manufacturing technology; through the principle of layer-by-layer manufacturing, a 3D computer model is printed as a 3D entity [1]. The advent of $3 \mathrm{D}$ printing has changed the traditional production and manufacturing model, simplifying complex production processes. 3D printing has the advantages of greater material saving and cost reduction over the traditional method of reducing material manufacturing. It can also solve the difficult processing problem of complicated structure parts. It is an ideal processing method specifically for hard and brittle ceramic material parts. 3D printing technology, along with digital modeling, materials technology, and other areas of cutting-edge technology, has profoundly changed the process and methods of object manufacturing. Recognized as "the most iconic production tool for the third Industrial Revolution," 3D printing has gained increasing attention both at home and abroad $[2,3]$.

In 3D printing, the printing of resin and metal materials is a mature technology. Yuki Nakagawa et al. [4] researched 3D printing of carbon fiber-reinforced plastic parts. Carbon fiber-reinforced plastic parts can be manufactured by sandwiching carbon fibers between upper and lower $A B S$ layers made by a 3D printer using fused deposition modeling (FDM). The strength is increased not only by sandwiching the carbon fibers but also by thermal bonding between the fibers and layers. The strength for a smalldiameter nozzle is higher than that for a large-diameter nozzle. Developing advanced production processes by using 3D printing is desirable. Domínguez-Rodríguez et al. [5] filled cylindrical specimens with honeycomb and rectangular patterns to test $3 \mathrm{D}$ printed $\mathrm{ABS}$ structures under

$\triangle$ Hongchao Ji, jihongchao@ncst.edu.cn | ${ }^{1}$ National Center for Materials Service Safety, University of Science and Technology Beijing, Beijing 100083, China. ${ }^{2}$ School of Mechanical Engineering, North China University of Science and Technology, 21 Bohai Road, Caofeidian Xincheng, Tangshan 063210, Hebei, China. ${ }^{3}$ National Capital Office, North China University of Science and Technology, Tangshan 063210, China. ${ }^{4}$ College of Mechanical and Electrical Engineering, Hebei Agricultural University, Baoding 071001, China. 
compressive loads to analyze the role of printing direction, density, printing time, and filler shape in stiffness, strength, and failure mechanisms. Results show that the rectangular filler shape is suitable for fast mass production systems. 3D printed structures filled with honeycomb patterns in the longitudinal direction have the most effective strength/mass ratio and are thus recommended for low-weight applications. A rectangular or honeycomb pattern with $100 \%$ relative density structures exhibits the highest strength recommended for applications where weight is not limited. Travitzky [5] offers a review of present achievements in the field of processing of ceramicbased materials with complex geometry using the main additive manufacturing (AM) technologies. Wu [6] report a novel approach to fabricate dense zirconia-toughened alumina (ZTA) ceramics with excellent properties via an additive manufacturing process based on stereolithography. Compared with metal and resin material printing technology, ceramic material additive manufacturing is a relatively new technology [2].

Precursor ceramic materials have excellent properties of high hardness, high strength, low density, high temperature resistance, and corrosion resistance. They have broad application prospects in high-tech fields, such as aviation, aerospace, electronics, and nuclear energy, as well as general industrial fields, such as machinery, metallurgy, and chemical industry [7-9]. At present, the 3D printing technology for ceramic materials mainly includes inkjet printing (IJP) [10], stereo lithography [6, 11], laminated object manufacturing [12-14], selective laser sintering [15, 16], $3 D$ printing $[17,18]$, 3D gel-printing (3DGP) [19], and FDM $[20,21]$. FDM is a typical representative of additive manufacturing technology based on the extrusion process. The advantages of FDM are its low cost, wide range of materials, easy process control, and the ability to create complexshaped parts.

In recent years, for ceramic materials in 3D printing extrusion research, researchers have emphasized the molding process. Shao et al. [22] employed the 3DGP process by using a screw extruder to prepare zirconia ceramic parts with a regular appearance; the surface roughness of the printed ceramic green sample is $8.9 \mu \mathrm{m}$. Zhang et al. [23] applied the 3DGP process to prepare hydroxyethyl methacrylate parts with WC-20Co solid content of $47-56$ vol\% by using a screw extruder. Samples with a good shape and a homogeneous microstructure can be printed with appropriate precision. Xu et al. [24] found that hydroxyapatite artificial bones successfully prepared by using the FDM process have natural bone mechanical properties and good in vitro cell biocompatibility. FDM is a simple, convenient, and relatively low-cost method. Liu et al. [25] analyzed a piston extrusion device in terms of extrusion pressure, layer thickness, printing speed, and other process parameters on the basis of the printing results of alumina ceramic slurry, and prepared ceramic parts with a smooth surface and high precision.

Organic pioneer synthesis of ceramic materials technology originated in the 1960s. This technology has become a new method for preparing ceramic materials due to its advantages in terms of molecular scale design, net size forming, low pyrolysis temperature, and high temperature performance. The core process for the use of organic precursors (e.g., polycarbosilane, polysilane, and polysiloxane) is by first conducting pyrolysis preparation of ceramic materials, including organic small molecules, followed by condensation reaction into organic macromolecules, and then further cross-linking the molecules to create an organic-inorganic precursor. A ceramic material is then created after pyrolysis and sintering [26-28]. At present, the main types of ceramic materials synthesized by $3 \mathrm{D}$ printing combined with organic precursors include $\mathrm{SiC}, \mathrm{Si}_{3} \mathrm{~N}_{4}, \mathrm{SiOC}$, and SiNC [29]. Schaedler et al. [30] used organic polymer-based UV-curable 3D printing technology to obtain a polymer precursor with a 3D structure. At $1000{ }^{\circ} \mathrm{C}$, pyrolysis obtained almost the same shrinkage and high-density ceramic materials. This type of ceramic material has higher compressive and shear strength than ordinary ceramic foam with the same density. Pierin et al. [31] found that on the basis of the IJP process, prepared fine porous $\mathrm{SiO}$ ceramic scaffolds with preceramic polymer of precursors have good compressive strength. However, no researcher has applied precursor ceramic slurry to the extrusion of 3D printing.

Slurry extrusion device in 3D printer is the core component. This device delivers stock accurately and quantitatively. However, studies on slurry extrusion structure remain relatively few. Zhou et al. [32] created four different designs of the internal flow passage geometry of the plunger extruder and used fluid analysis software to simulate flow velocity and internal pressure field at the nozzle outlet cross section. The best shape of internal flow channel was obtained, but this study focused only on the plunger extruder. Ding et al. [33] improved the ceramic 3D printer nozzle structure to achieve continuous feeding and simulated the screw extrusion process, but the outflow rate is too small, and the flow rate may affect printing efficiency. Drotman [34] designed the 3D printer screw extrusion, which can achieve $0.2 \mathrm{~mm}$ nozzle diameter extrusion with a maximum outflow rate of $7.14 \mathrm{~mm} / \mathrm{s}$. However, resin material was used in this study.

This paper, which is based on experiments and numerical simulations, selected the FDM process based on extrusion technology as the technical basis and selected the ceramic precursor as print material. Two different extrusion methods-plunger and screw extrusion-combined with simulation analysis were used to finish the printing 
Fig. 1 Preparation of materials

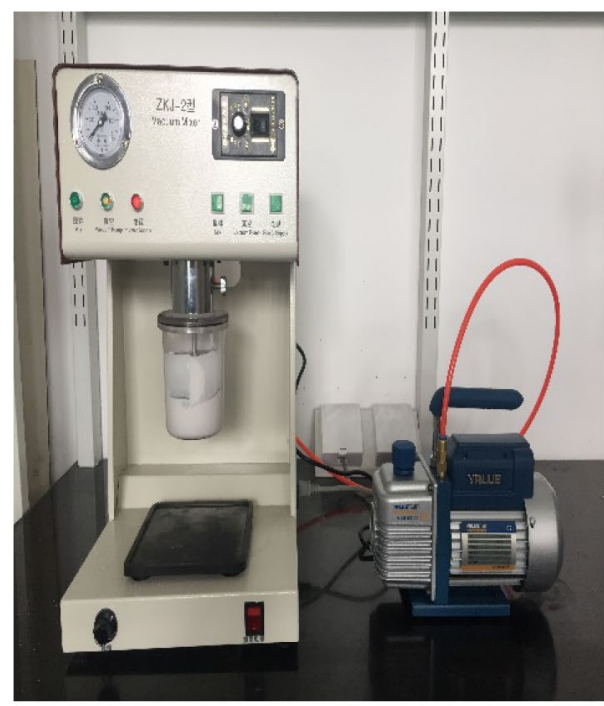

(a) Vacuum agitator

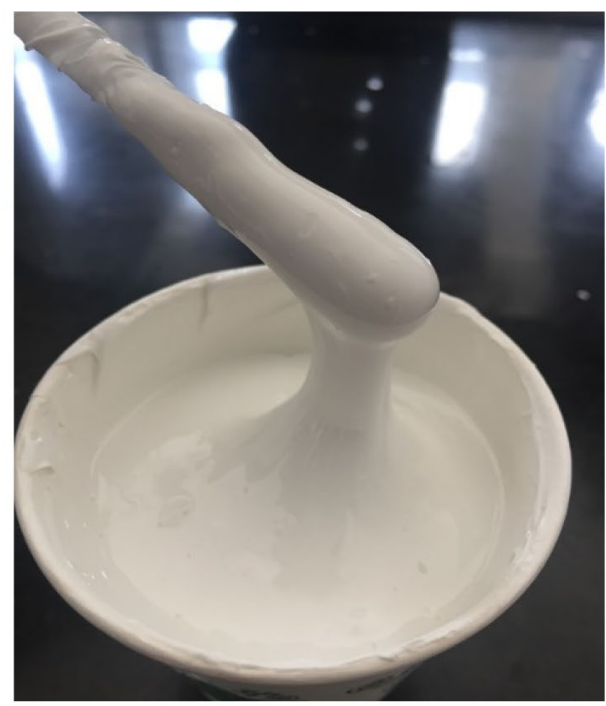

(b) Precursor ceramic slurry experiment by comparing the quality of the ceramic green samples printed by the two slurry extruders. The analysis aims to determine the best slurry extrusion device for precursor ceramic material 3D printer. Finally, the device is further improved and optimized based on the determined extruding device, which effectively improves the printing efficiency of the 3D printer.

\section{Experimental}

\subsection{Material}

Hydrosilicone oil, as a cross-linking agent, is added to vinyl silicone oil. Then, the appropriate amount of chloroplatinic acid and methylbutynol is added. Chloroplatinic acid is used as a catalyst for the hydrosilylation reaction of vinyl silicone and hydrosilicone oils. Methylbutynol is used to inhibit premature solidification of the precursor ceramic slurry. Finally, calcined kaolin is gradually added to the previous mixture and uniformly stirred to obtain the precursor ceramic slurry. There are many bubbles in the prepared precursor ceramic slurry, which needs to be stirred in a vacuum mixer. Figure $1 \mathrm{a}$ is a vacuum mixer and Fig. $1 \mathrm{~b}$ is a precursor ceramic slurry.

The slurry exhibits distinct non-Newtonian fluid properties. To further grasp its nature, the relationship between shear rate and dynamic viscosity was tested by using a torque rheometer (Fig. 2).

Figure 3 shows the relationship between shear stress and rate of the slurry. As can be seen from Fig. 3, the shear stress is a non-zero constant when the shear rate is zero. This result indicated that the fluid is Bingham plastic fluid.

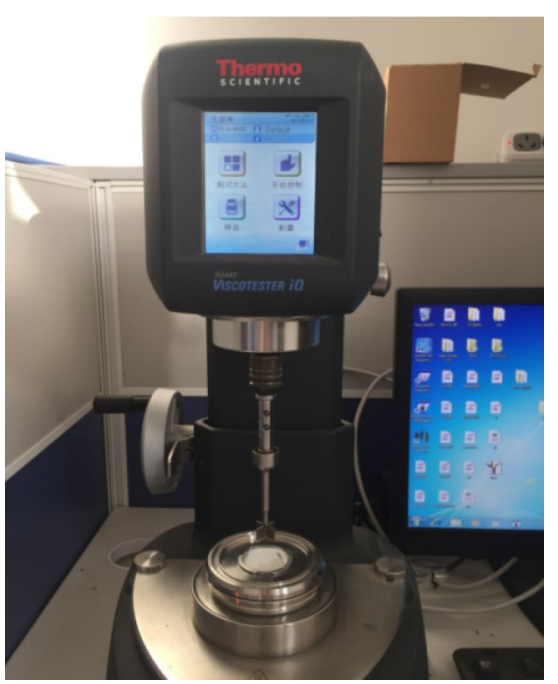

Fig. 2 Experimental torque rheometer

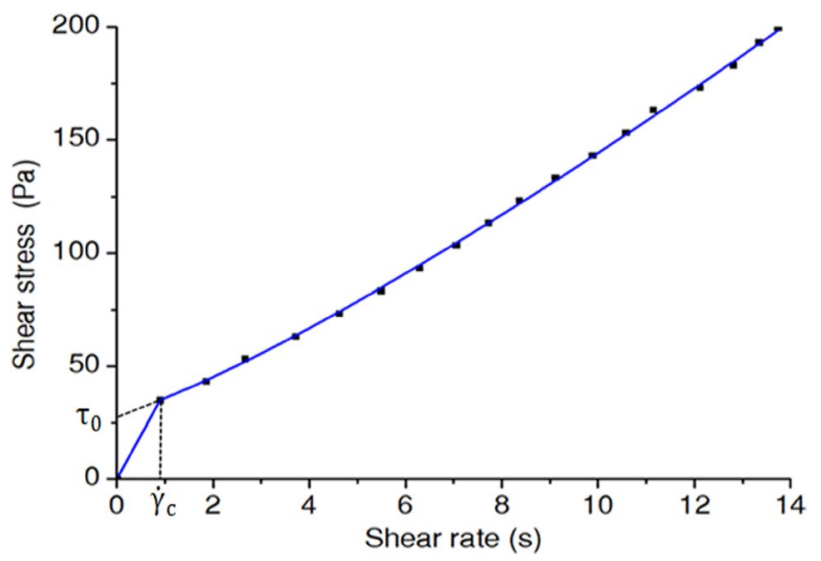

Fig. 3 Relationship between shear stress and rate 
The dynamic viscosity of the slurry with the shear rate changes in the relationship shown in Fig. 3. The curve fitting from Fig. 3 is

$\eta=\frac{28.5}{\dot{\gamma}}+7\left(\frac{\dot{\gamma}}{0.9}\right)^{0.21}$

Conforming to the Bingham plastic fluid viscosity formula,

$\eta=\frac{\tau_{0}}{\dot{\gamma}}+\mathrm{k}\left(\frac{\dot{\gamma}}{\dot{\gamma}_{c}}\right)^{\mathrm{n}-1}$

where $\eta$ is the dynamic viscosity; $\dot{\gamma}$ denotes the shear rate; $\tau_{0}$ represents the yield stress threshold; $\dot{\gamma}_{c}$ refers to the critical shear rate; $\mathrm{K}$ stands for the consistency index; and $\mathrm{n}$ is power law index. When $\mathrm{n}<1$, the fluid exhibits shear thinning. When $\mathrm{n}>1$, the fluid is shear thickening. When $\mathrm{n}=1$, the model is a Newtonian fluid rheological model.

The formula obtained from the fitted curve shows that $n=1.21$, indicating that the slurry has shear thickening properties; the yield stress threshold value is $28.5 \mathrm{~Pa}$. The critical shear stress value is $0.9(1 / \mathrm{s})$; the maximum and minimum viscosity values are 35.9 and $14.7 \mathrm{~Pa}$ s, respectively. Thus, the experimental printing slurry is a high-viscosity non-Newtonian fluid (Fig. 4).

\subsection{Plunger extruder}

Figure 5 shows a schematic of the 3D printing plunger extruder. The printing process is as follows: first, the ceramic slurry is gradually filled into the silo, and the plunger is installed. Commands are sent to the motor on the plunger via the printer control device to move the plunger down into the silo so that a small amount of slurry in the silo overflows. This process can prevent too much air from entering the silo during feeding and reducing

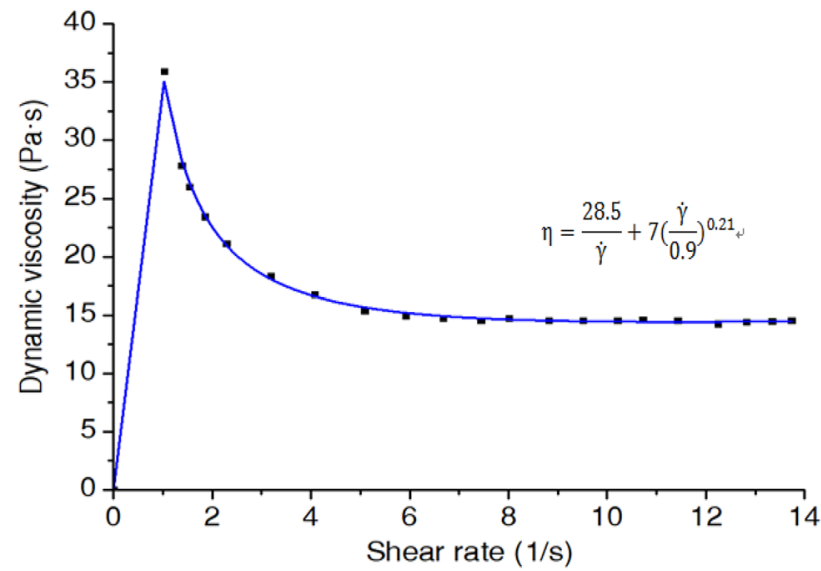

Fig. 4 Relationship between dynamic viscosity and shear rate

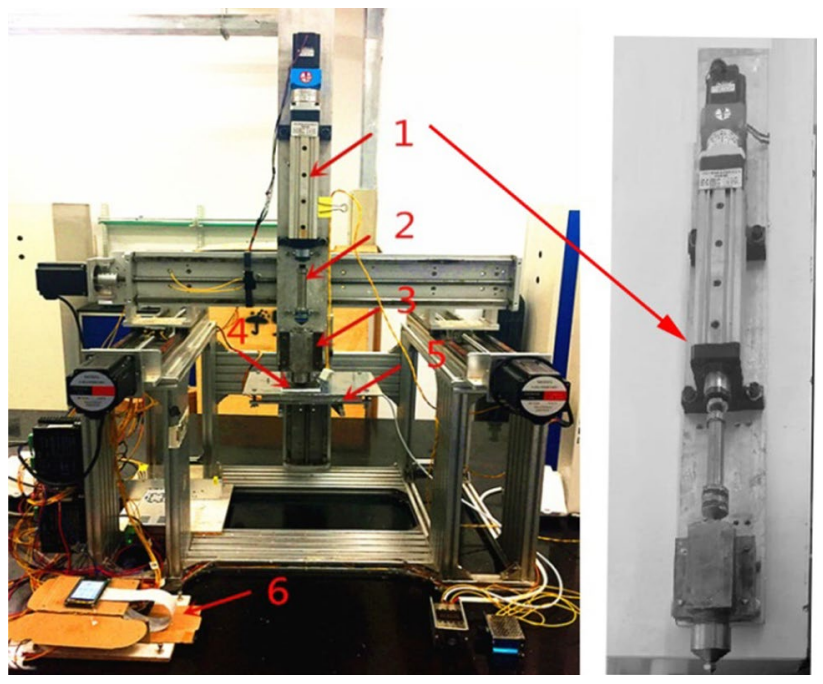

Fig. 5 Plunger extruder. 1, Motor; 2, plunger; 3, silo; 4,5, heating device; 6 printer control device

printing accuracy. Second, the designed 3D model is imported into the slicing software, which can rotate the imported model to the most suitable printing angle and set reasonable printing parameters. Print speed, layer height, and nozzle diameter are key parameters that affect print quality; a nozzle diameter of $0.6 \mathrm{~mm}$, a layer height of $0.4 \mathrm{~mm}$, and a print speed of $11 \mathrm{~mm} / \mathrm{s}$ can generate high printing accuracy for the precursor ceramic material parts. The slice is layered to generate $\mathrm{G}$ code and imported into the 3D printer control device. Finally, driven by the motor, the plunger moves downward to produce a significant pressure bin, forcing the slurry to flow from the nozzle and through the heating device to achieve curing.

\subsection{Screw extruder}

The printing results of the screw and plunger extruders, as experimental and control groups, respectively, are compared to determine the best slurry extrusion for the precursor ceramic 3D printer. Figure 6 shows a schematic of the 3D printing screw extruder. First, the silo is filled with the precursor ceramic slurry, then the piston with the diameter of the silo is pressed into the silo, and the air is discharged through repeated vibration bin. With access to the upper opening of the silo plastic pipe, the plastic pipe is connected to the pressure gauge and air compressor. The plastic pipe connected to the bottom outlet allows slurry to enter the screw extruder. The air compressor is opened when printing, and gas pressure is controlled through the pressure gauge reading so that the piston in the silo will generate thrust to ensure that the slurry will always fill the screw device without flowing out of the nozzle. The $\mathrm{G}$ code is imported into the 3D printer control 


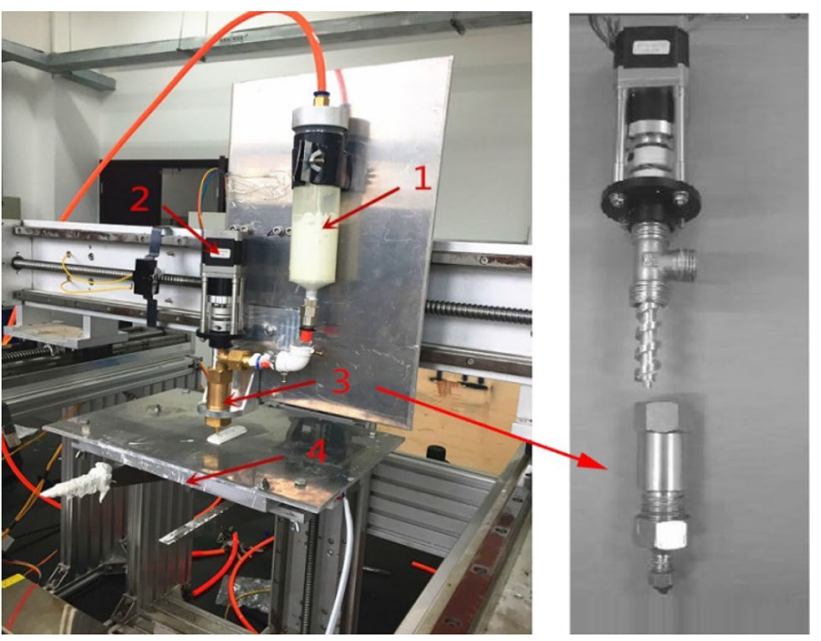

Fig. 6 Screw extruder. 1, Silo; 2, motor; 3, screw; 4, heating device

device, where the 3D model and the printing parameters are consistent with the plunger extrusion printing. Finally, driven by the motor, the screw rotation generates axial force to realize slurry transportation, which causes the slurry to flow out of the nozzle and solidify through the heating device.

\section{Finite element model}

In this paper, the extrusion process of the plunger and screw extruders are simulated by using ANSYS Fluent software. The experiment used precursor ceramic slurry as a variable density fluid, the reference pressure is standard atmospheric pressure, the density at reference pressure is $1538 \mathrm{~kg} / \mathrm{m}^{3}$, and the density exponent of the slurry is 716 . The Herschel-Bulkley model was used as the rheological model. Extrusion molding was performed at room temperature. The extrusion molding process was evaluated as a laminar flow in accordance with the Reynolds number formula.

First, the finite element model of the plunger extruder is established. The model is divided by the hexahedral structure grid. The total number of grids is 929,998 . Figure 7 presents the plunger extruder structure model. Figure 8 shows the plunger extruder grid model. In accordance with the printing experiment, the plunger moves downward with speed when squeezing the slurry; the inlet velocity is set as $12 \mathrm{~mm} / \mathrm{s}$. In accordance with the actual situation, the slurry flows directly into the atmosphere; the outlet pressure value is set as $0 \mathrm{~Pa}$.

The finite element model of the screw extruder established. The model is divided by a total of 2658,133 structural and unstructured grids. Figure 9 presents the screw

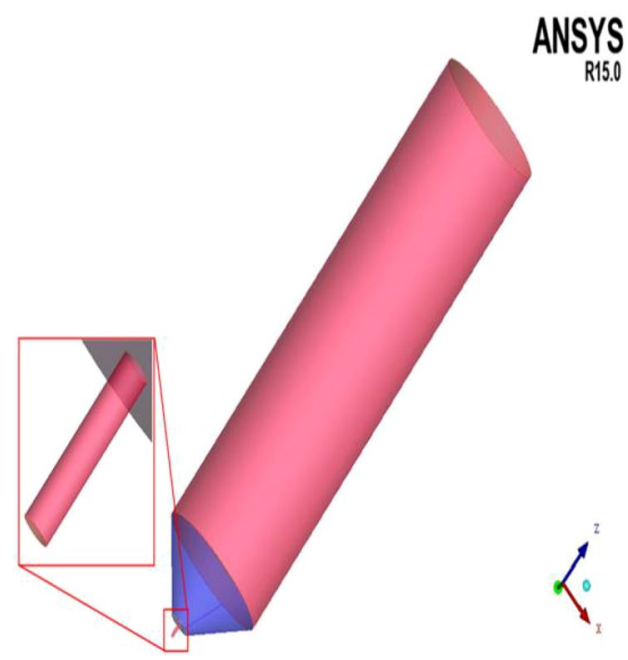

Fig. 7 Plunger extruder structure model

extruder structure model, and Fig. 10 shows the screw extruder grid model.

\section{Results and discussion}

\subsection{Comparison of experimental results}

Figure 11 presents the result of the ceramic green sample printed by the plunger extruder. Figure 11 shows that the ceramic green sample printing line has slight ripples on the sidewall surface, which may be caused by the large or uneven slurry discharge during the printing process. From the front, the printing lines at both ends of the ceramic green sample are connected, which is difficult to observe

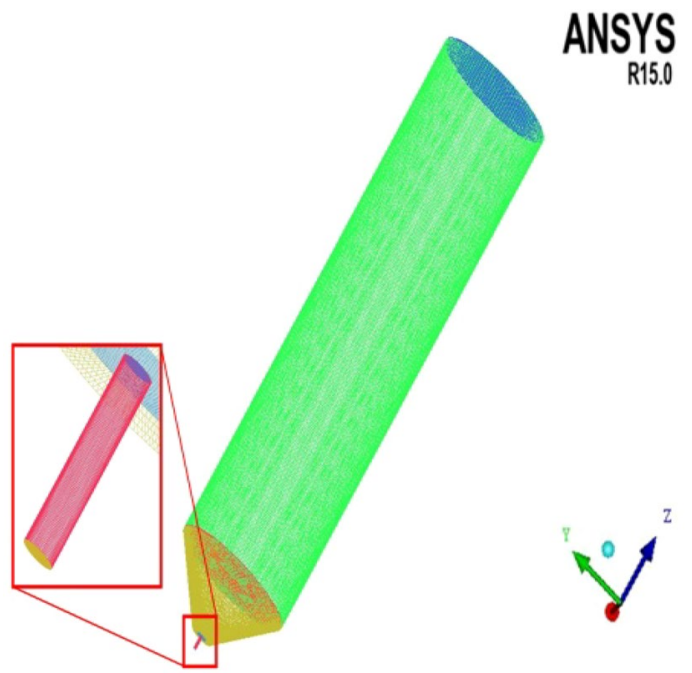

Fig. 8 Plunger extruder grid model 


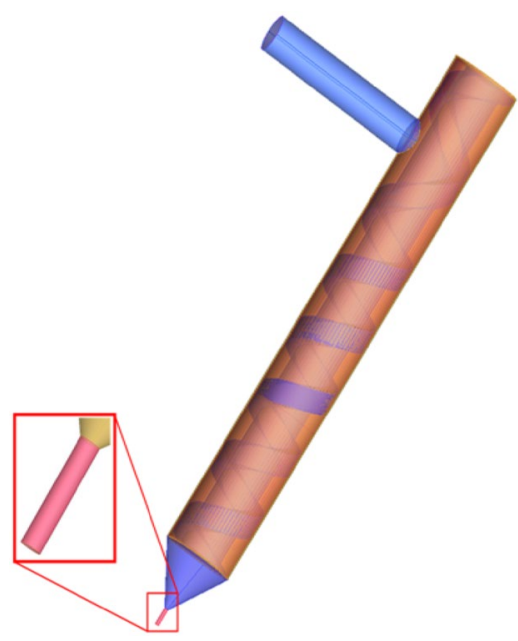

$\underset{\text { R15.0 }}{\text { ANSYS }}$

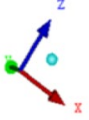

Fig. 9 Screw extruder structure model

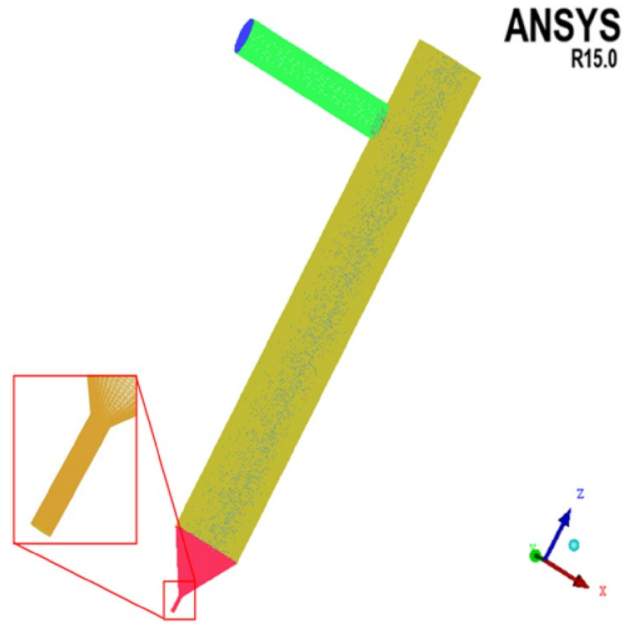

Fig. 10 Screw extruder grid model

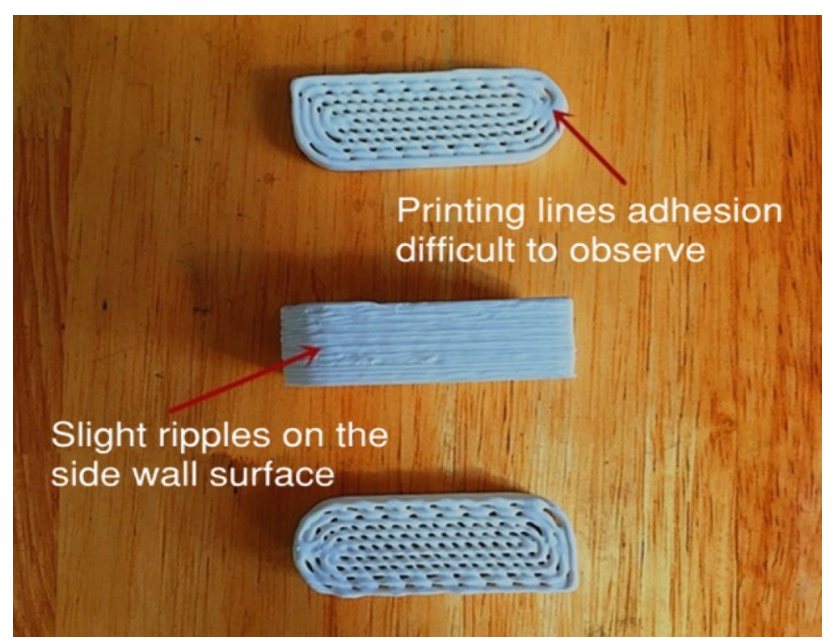

Fig. 11 Plunger extruder-printed ceramic green sample

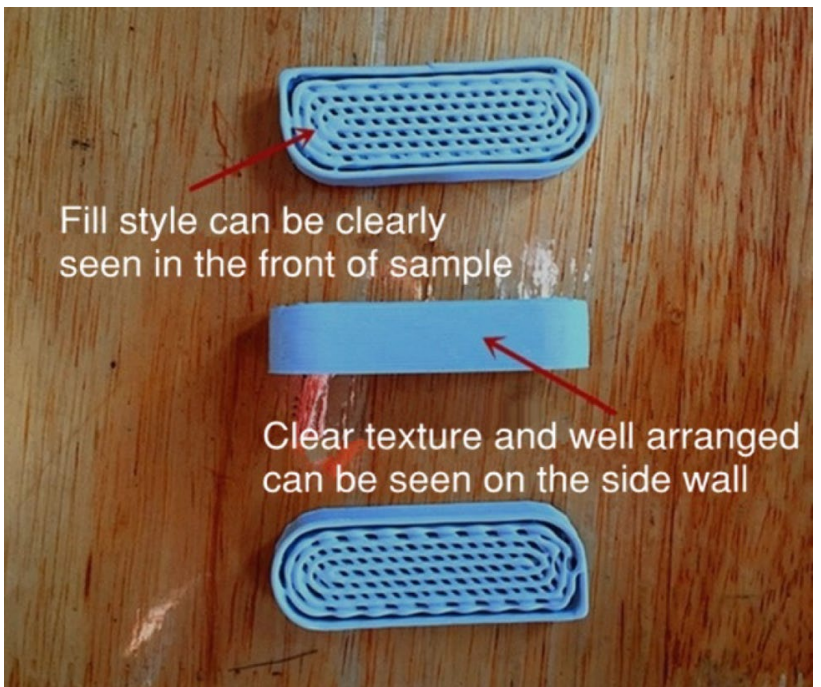

Fig. 12 Screw extruder-printed ceramic green sample

and is also slightly protruding, thereby indicating that the discharge volume at this part is larger than that at other parts. Therefore, the surface of the ceramic green sample will no longer be flattened when the number of printing layers is high, and the two ends of the green sample will be higher than the middle height, resulting in deformation.

Figure 12 shows the result of the ceramic green sample printed by the screw extruder. No defect or deformation can be observed outside the printed sample. The sidewall of the ceramic sample has a clear texture and is well arranged. The filling process in the printing style can be distinctly seen at the front of the printing sample. Thus, the printing effect of the screw extruder on the ceramic green sample during the uniform and stable discharge process is significantly better than that of the plunger extruder.

\subsection{Simulation result analysis}

When printing two ends of the ceramic green sample, the printing speed reduces due to the change in printing direction at the turn. To ensure uniform outlet velocity, the plunger stops moving down briefly and the screw stops rotating. In the ideal state, slurry should have stopped flowing out. The ceramic slurry is a compressible liquid. Thus, extrusion pressure allows the slurry to compress to a certain extent, and internal pressure is accumulated. When the external force is removed, under the effect of accumulated pressure, the slurry in the silo still flows out from the printing head, thereby causing the "salivation" phenomenon, which commonly occurs in the 3D printing process and causes the surface of ceramic green sample to bulge due to overfilling [35]. High thickness of the printed ceramic green sample corresponds to an uneven surface.

\section{SN Applied Sciences}


Figure 13 shows the simulated changes in the average flow velocity at the outlet of the plunger and screw extruder as the print orientation changes. At $0.05 \mathrm{~s}$ before the print speed changes, the average outlet velocities of the plunger and screw extruders reached a steady state. The average outlet velocities of the plunger and screw extruders are $10.83 \mathrm{~mm} / \mathrm{s}$ and $10.81 \mathrm{~mm} / \mathrm{s}$, respectively.

The plunger then stopped moving, but the average flow velocity at the outlet did not decrease; instead, it increased slightly. The screw extruder is different; the average outlet velocity after the screw stopped rotating decreased from 10.81 to $2.25 \mathrm{~mm} / \mathrm{s}$ in only $0.23 \mathrm{~s}$, which quickly controlled the discharge volume.

Finally, the printing direction completely changed, the plunger moved back down, and the average flow velocity at the outlet still exhibited a slight increase, which explains the small ripple on the sidewall surface of the printed ceramic green sample due to the relative discharge. The corresponding screw to restore rotation, this time if the average outlet velocity cannot be restored to the level before the screw stall there will be too little due to filling the slurry caused by the problem of depression of the print model. In accordance with the results, the printed ceramic green sample does not appear to be depressed due to the small amount of discharged material. Simulation results show that the average velocity at the outlet increased to $10.7 \mathrm{~mm} / \mathrm{s}$ in only $0.1 \mathrm{~s}$, which is equivalent to the average flow velocity at the outlet before the screw stopped. This result shows that the screw extruder can quickly control the amount of output to match the needs of 3D printing.

In the $0.05-0.32 \mathrm{~s}$ time period, the area between the plunger extruder and the average extrusion rate at the outlet of the screw extruder represents the excess discharge of the plunger extruder over the screw extrusion device during a change in print orientation. This activity

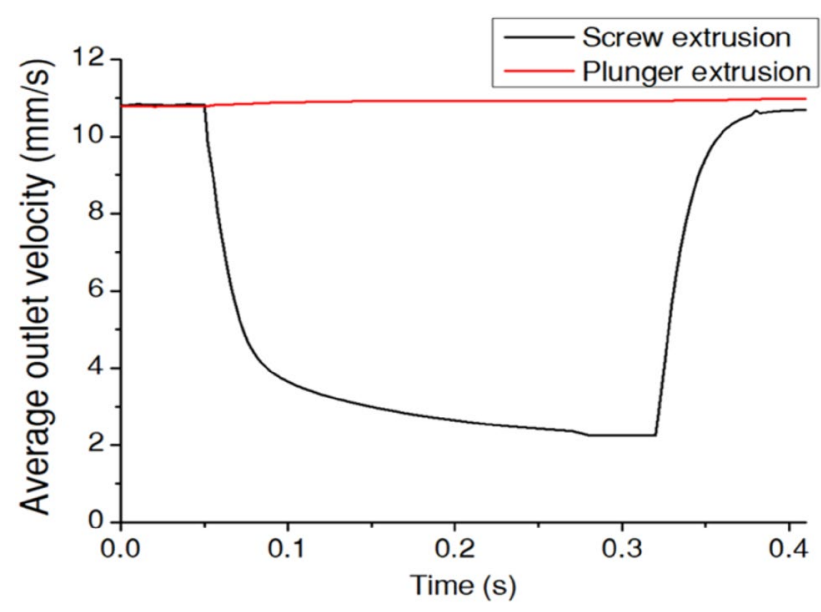

Fig. 13 Average outlet velocity comparison chart is an important reason why the print lines on both ends of the ceramic green sample connected and bulged and why the printing effect of the screw extruder is significantly better than that of the plunger extruder. The area between the flow velocity at the outlet of the screw extruder and the time axis ( $\mathrm{X}$-axis) represent the excess discharge of the screw extruder in the printing direction. These extra discharges are sufficient to significantly affect print accuracy. Thus, the screw extruder can print a high-quality ceramic green sample.

Figure 14 presents a comparison diagram of printing direction changes before and after the change in the pressure of the plunger and screw extruders. Figure 14a, $c$, and e show the description of the pressure contour of the plunger extruder before the print orientation changes, $0.23 \mathrm{~s}$ after the plunger stops moving, and $0.1 \mathrm{~s}$ after the print orientation changes, respectively. As can be seen from the abovementioned figure, the pressure value of the cross section of the plunger extruder fundamentally exhibits no change, and the pressure value of the connected atmospheric pressure rapidly drops at the nozzle near the outlet. Before the printing direction changed, the pressure in the silo was $60,489.69 \mathrm{~Pa}$. After the plunger stopped moving at $0.23 \mathrm{~s}$, the average velocity at the outlet was stable, but the internal pressure of the silo decreased by only 593.99 $\mathrm{Pa}$. This outcome occurred because the plunger extruder of the silo is a confined space; when the plunger stops moving down, the pressure inside the silo was not substantially released. The release of small pressure in turn causes the slurry density to become small and expanded. Thus, the slurry will continue to flow from the nozzle and the velocity will not be reduced. When the printing direction is changed, the pressure in the silo increases to $61,142.33$ Pa within $0.1 \mathrm{~s}$. This increase may be due to the plunger moving down again to squeeze the slurry, thereby further increasing the outlet flow rate.

Figure $14 b, d$, and $f$ present the pressure contour of the screw extruder before the printing direction changes, $0.23 \mathrm{~s}$ after the screw stops rotating, and $0.1 \mathrm{~s}$ after the printing direction is changed, respectively. The abovementioned figure shows that screw extrusion is a significant pressurization process, which shows that the variable pitch screw can effectively compensate for the pressure at the inlet, which is not enough to ensure sufficient pressure to achieve slurry extrusion. Before the printing direction changed, the screw extruder internal pressure changes from 10,000 to $59,786.29 \mathrm{~Pa}$. After the screw stopped rotating at $0.23 \mathrm{~s}$, the internal pressure of the screw extruder dropped from $59,786.29$ to $10,000 \mathrm{~Pa}$, which was equivalent to the inlet pressure. This decrease shows that the pressure generated by the screw has been released, which corresponds to a significant reduction in the outlet velocity. When the printing direction changed, the screw 
Fig. 14 Comparison diagram of printing direction changes before and after the pressure of the plunger and screw extruders changes. a Printing direction changes before the plunger extruder pressure contour; b printing direction changes before the screw extruder pressure contour; $\mathbf{c}$ the plunger stopped moving after $0.23 \mathrm{~s}$ of pressure contour; $\mathbf{d}$ the screw stopped rotating after $0.23 \mathrm{~s}$ of pressure contour; e printing direction changes after $0.1 \mathrm{~s}$ plunger extruder pressure contour; f printing direction changes after $0.1 \mathrm{~s}$ screw extruder pressure contour (a)

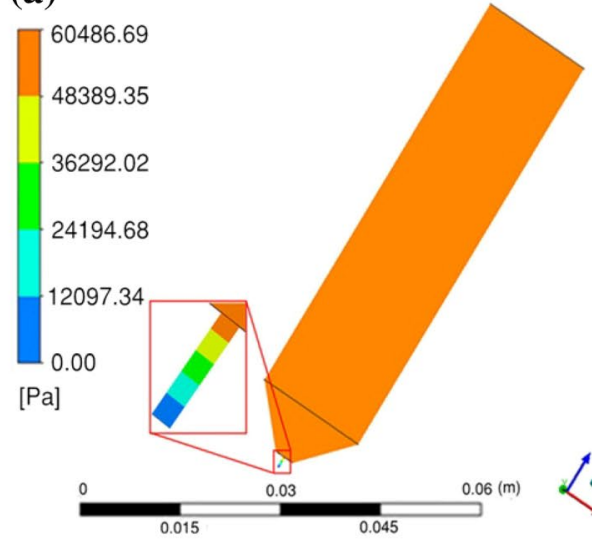

(c)

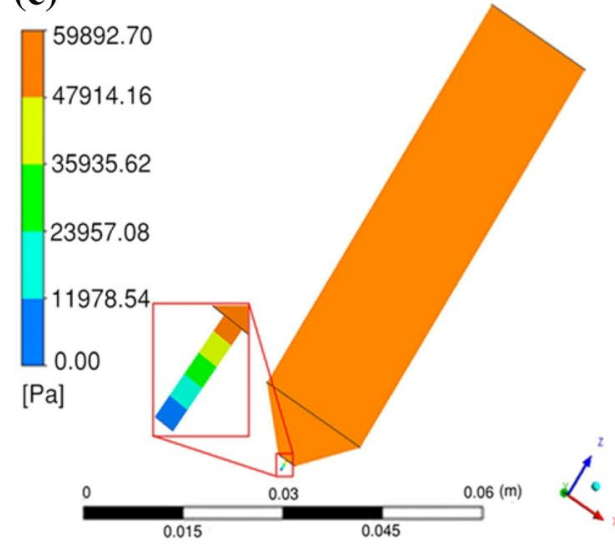

(e)

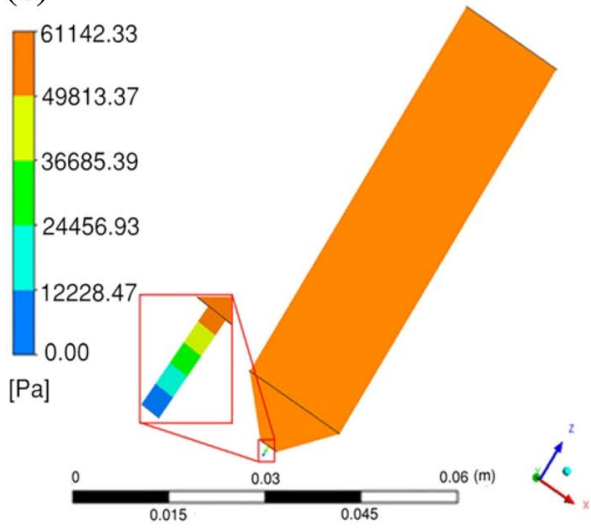

(b)

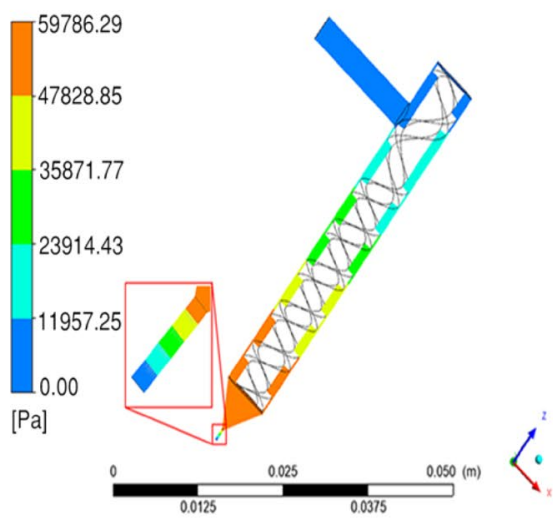

(d)

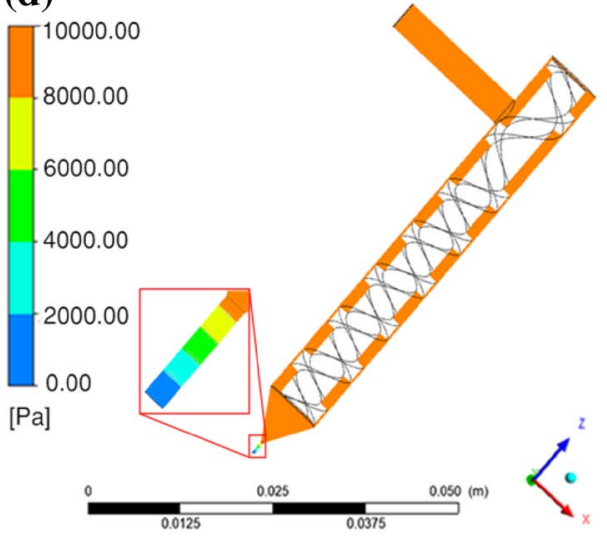

(f)

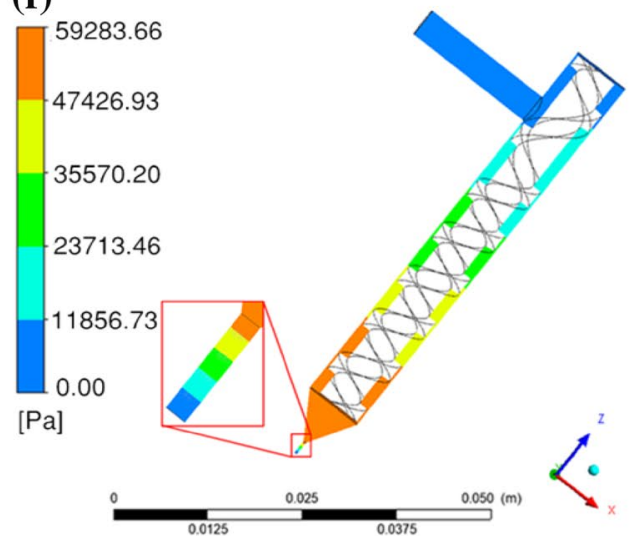

resumed rotation. In only $0.1 \mathrm{~s}$, the internal pressure of the screw extruder rose to $59,263.66 \mathrm{~Pa}$, which is equivalent to the internal pressure of the device before the screw is stopped. This result is an important reason for the rapid increase in the outlet velocity.

\subsection{Improvement and optimization}

Earlier studies identified screw extruders as the best extrusion device for 3D printers when using precursor ceramic materials. The ceramic green sample printed by the screw extruder has high precision and good surface quality. However, the printing speed is only $11 \mathrm{~mm} / \mathrm{s}$, and the low printing speed affects printing efficiency, which is caused by the low average velocity at the printer outlet. To shorten the printing time and improve printing efficiency, the change in the screw speed to 60 and $90 \mathrm{rpm}$ is first simulated by the finite element method. Then, the change in the average outlet velocity is observed to improve the printing efficiency under the premise of ensuring printing quality. Figure 15 shows the change in the average outlet velocity at screw speeds of 40,60 , and $90 \mathrm{rpm}$ when the 


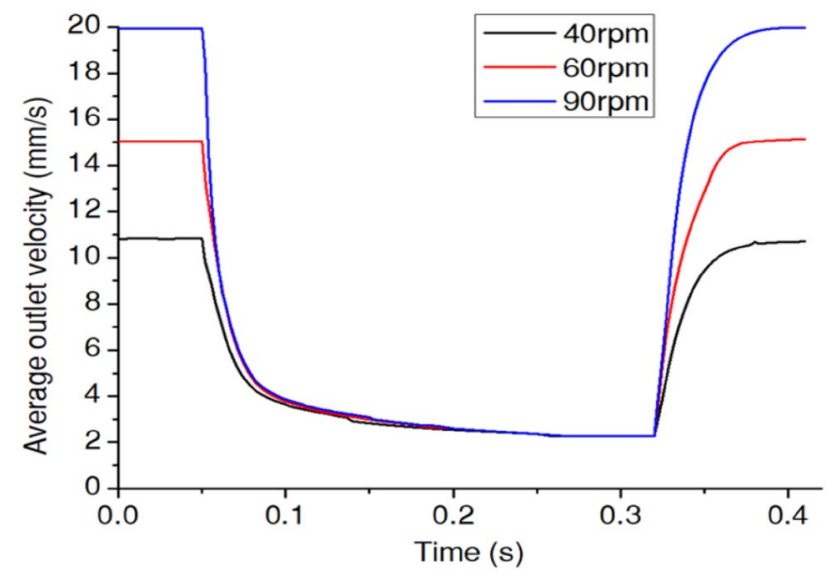

Fig. 15 Change in average outlet velocity at different screw speeds when the printing direction changed

printing direction changed. The abovementioned figure shows that when the screw speed increases, the average outlet velocity also increases, corresponding to an increase in the printing speed. When the printing direction changes, the screw extruder can still control the discharge volume quickly, and the printing quality will not be reduced due to the change in the rotation speed. When the screw speed reaches $90 \mathrm{rpm}$, the average outlet velocity can reach up to $19.98 \mathrm{~mm} / \mathrm{s}$, which is nearly double that of the outlet before optimization.

When the screw speed is adjusted to $90 \mathrm{rpm}$ and the printer stepper motor of each axis is adjusted to match the print speed, the printing speed increases from $11 \mathrm{~mm} / \mathrm{s}$ to $20 \mathrm{~mm} / \mathrm{s}$, and the optimized 3D printing experiment is conducted. Figure 16 shows the ceramic green samples printed at a screw speed of $90 \mathrm{rpm}$. The printed ceramic green sample had a smooth surface and no defects and
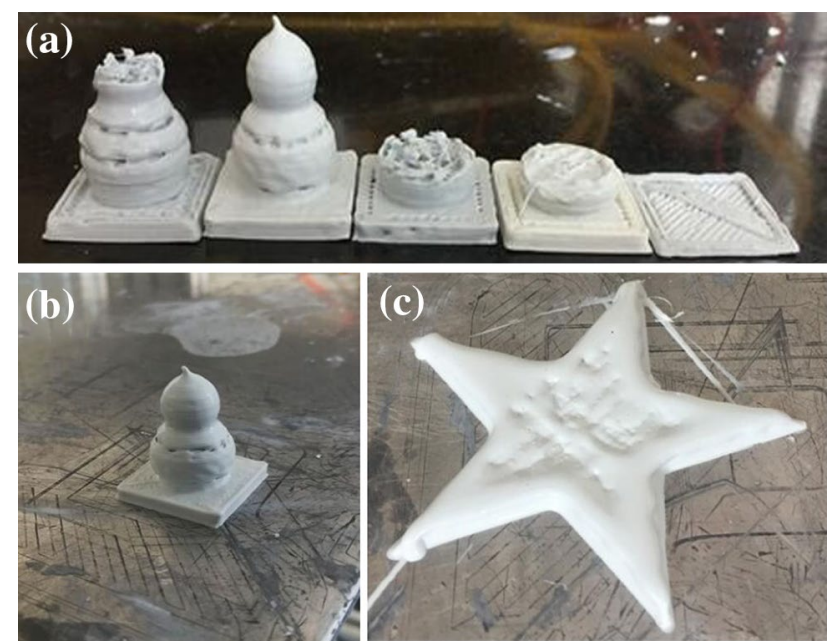

Fig. 16 Ceramic green sample printed with screw speed of $90 \mathrm{rpm}$ deformation. Moreover, the printing speed improved significantly. Thus, increasing the screw speed ensures that the precursor ceramic green sample is efficiently printed while meeting printing accuracy requirements.

Figure 17 shows the microstructure distribution of the ceramic samples. The surface of the samples are flat. Because of the high viscosity and poor fluidity of printing slurry, there are bulky protuberances and inhomogeneous phenomena on the surface of cured slurry.

\section{Conclusion}

In this paper, FDM process based on extrusion technology was selected as the technical basis for the successful printing of the precursor ceramic material green sample. First, two different extrusion methods-plunger and screw extrusion-were used to shape the ceramic green sample. The quality of the extrusion methods were compared, and a simulation analysis was conducted. Finally, the screw extruder was optimized. The following conclusions were obtained:
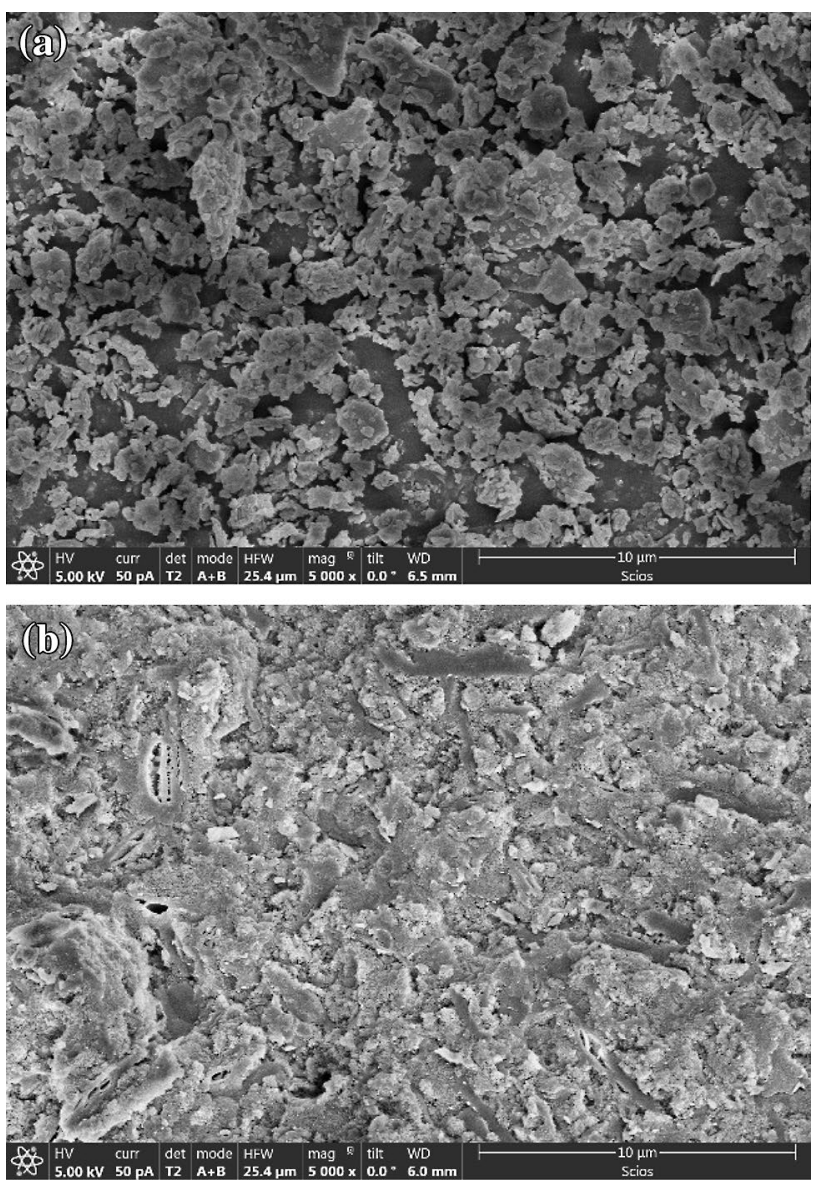

Fig. 17 Microstructure distribution 
1. Experiments found that the ceramic green sample printed by the plunger extruder has slight ripples on the sidewall surface.And the ceramic green sample printed by the screw extruder has a smooth surface, a clear texture, and a distinct layer, and the print quality is significantly superior to that of the plunger extruder.

2. The simulation analysis shows that when the printing direction changes, the screw extruder can quickly and accurately control the amount of discharge, whereas the plunger extruder exhibited almost no change in the amount of discharge. Therefore, the ceramic green sample printed by the screw extruder is more planar and has higher molding precision than that printed by the plunger extruder.

3. Increasing the screw speed can significantly improve print efficiency without affecting print quality. When the screw speed is $40 \mathrm{rpm}$, the printing speed is $11 \mathrm{~mm} / \mathrm{s}$; when the screw speed is $90 \mathrm{rpm}$, the printing speed increases to $20 \mathrm{~mm} / \mathrm{s}$. At this time, the screw extruder can still control the increase and decrease of the discharging amount rapidly, thus ensuring printing precision.

Acknowledgements This work is supported by the National Natural Science Foundation of China (Grant No. 51505124). This work is also supported by the National Natural Science Foundation of China (Grant No. 51375042).

\section{Compliance with ethical standards}

Conflicts of interest The authors declare no conflict of interest.

\section{References}

1. Liu N, Guo H, Fu L et al (2008) Three-dimensional photonic metamaterials at optical frequencies. Nat Mater 7(1):31-37

2. Zocca A, Colombo P, Gomes CM et al (2015) Additive manufacturing of ceramics: issues, potentialities, and opportunities. J Am Ceram Soc 98(7):1983-2001

3. Nakagawa Y, Mori KI, Maeno T (2017) 3D printing of carbon fibrereinforced plastic parts. Int J Adv Manuf Technol 91:1-7

4. Domínguez-Rodríguez G, Ku-Herrera JJ, Hernández-Pérez A (2017) An assessment of the effect of printing orientation, density, and filler pattern on the compressive performance of $3 D$ printed $A B S$ structures by fuse deposition. Int J Adv Manuf Technol 2:1-11

5. Travitzky N, Bonet A, Dermeik B et al (2014) Additive manufacturing of ceramic-based materials. Adv Eng Mater 16(6):729-754

6. Wu H, Liu W, He R et al (2017) Fabrication of dense zirconiatoughened alumina ceramics through a stereolithographybased additive manufacturing. Ceram Int 43(1):968-972

7. Huang $M, W u H$, Huang $R$ et al (2017) Research progress in ceramic additive manufacturing (3D printing) technology. Adv Ceram 4:248-266

8. Miao H, Lin X, Qi L (2008) Research progress of advanced structural ceramics. Rare Mater Eng 37(a01):14-19
9. Xiong L, Xu Y (2007) Ceramic precursor polymer applications. Progr Chem 19(4):567-574

10. Ken'Ichi K (2012) Recent advanced ink jet printing technology. Pigments Paints Print Inks 85(6):254-258

11. Song X, Chen Z, Lei L et al (2017) Piezoelectric component fabrication using projection-based stereolithography of barium titanate ceramic suspensions. Rapid Prototyp J 23(1):44-53

12. Tumbleston JR, Shirvanyants D, Ermoshkin N et al (2015) Additive manufacturing. Continuous liquid interface production of 3D objects. Science 347(6228):1349-1362

13. Zheng DG (2013) About laminated object manufacturing technology research status report. Hunan Agric Mach 9:90-91

14. Zhang $\mathrm{Y}, \mathrm{He} \mathrm{X}$, Du S et al (2001) $\mathrm{Al}_{2} \mathrm{O}_{3}$ ceramics preparation by LOM (laminated object manufacturing). Int J Adv Manuf Technol 17(7):531-534

15. Shishkovsky I, Nagulin K, Sherbakov V (2015) Laser sinterability and characterization of oxide nano ceramics reinforced to biopolymer matrix. Int J Adv Manuf Technol 78(1-4):449-455

16. Chen AN, Wu JM, Liu K et al (2017) High-performance ceramic parts with complex shape prepared by selective laser sintering: a review. Adv Appl Ceram 4:1-18

17. Bergmann C, Lindner M, Zhang W et al (2010) 3D printing of bone substitute implants using calcium phosphate and bioactive glasses. J Eur Ceram Soc 30(12):2563-2567

18. Suwanprateeb J, Sanngam R, Suvannapruk W et al (2009) Mechanical and in vitro performance of apatite-wollastonite glass ceramic reinforced hydroxyapatite composite fabricated by 3D-printing. J Mater Sci Mater Med 20(6):1281-1289

19. Ren $X$, Shao H, Lin T et al (2016) 3D gel-printing-An additive manufacturing method for producing complex shape parts. Mater Des 101:80-87

20. Castro J, Rojas-Nastrucci EA, Ross A et al (2017) Fabrication, modeling, and application of ceramic-thermoplastic composites for fused deposition modeling of microwave components. IEEE Trans Microw Theory Tech 65(6):2073-2084

21. Xu W, Du J, Chen X (2017) Ceramic 3D printing process of choice. Mech Electr Eng Technol 8:108-111

22. Shao H, Zhao D, Lin T et al (2017) 3D gel-printing of zirconia ceramic parts. Ceram Int 43:13938-13942

23. Zhang X, Guo Z, Chen G (2017) Additive manufacturing of WC20Co components by 3D gel-printing. Ceram Int 10:215-223

24. Xu N, Ye X, Wei D et al (2014) 3D artificial bones for bone repair prepared by computed tomography-guided fused deposition modeling for bone repair. ACS Appl Mater Interfaces 6(17):14952-14963

25. Liu J, Wu M, Cai J et al (2015) Effect of process parameters on the quality of 3D printed ceramic parts. J Shanghai Univ Electr Power 31(4):376-380

26. Schulz M, Börner M, Göttert J et al (2004) Cross linking behavior of preceramic polymers effected by UV and synchrotron radiation. Adv Eng Mater 6(8):676-680

27. Ma Q, Chen C, Zheng W et al (2004) Crosslinking and cleavage of polysiloxanes for ceramic precursors. Polym Mater Sci Eng 20(2):198-200

28. Ma Q, Chen C, Zheng W et al (2005) Study on crosslinking and cracking ceramics of polysiloxane. Polym Mater Sci Eng 21(2):279-282

29. Colombo P, Mera G, Riedel R et al (2010) Polymer-derived ceramics: 40 years of research and innovation in advanced ceramics. J Am Ceram Soc 93(7):1805-1837

30. Eckel ZC, Zhou C, Martin JH et al (2016) Additive manufacturing of polymer-derived ceramics. Science 351(6268):58-68

31. Pierin G, Grotta C, Colombo P et al (2016) Direct Ink writing of micrometric SiOC ceramic structures using a preceramic polymer. J Eur Ceram Soc 36(7):1589-1594 
32. Zhou J, Duan G, Lu L et al (2015) Study on key problems of microflow extrusion for ceramic. China Mech Eng 26(22):3097-3102

33. Ding C, Wu W, Zhu W et al (2017) Structure design and simulation analysis of ceramic 3D printer nozzle. JTianjin Polytech Univ 36(4):84-88

34. Drotman DTJ (2015) Design of a screw extruder for additive manufacturing. University of California, San Diego
35. Roopavath UK, Malferrari S, Van HA et al (2019) Optimization of extrusion based ceramic 3D printing process for complex bony designs. Mater Des 162:263-270

Publisher's Note Springer Nature remains neutral with regard to jurisdictional claims in published maps and institutional affiliations. 